Research Paper

\title{
Comparative Outcomes of Induction Chemotherapy Followed By Definitive Chemoradiotherapy versus Chemoradiotherapy Alone In Esophageal Squamous Cell Carcinoma
}

\author{
Li-Ling Luo1, 2, Mian Xi1, 2, Ya-Di Yang1,3, Qiao-Qiao Li11, 2, Lei Zhao1,2, Peng Zhang1,2, Shi-Liang Liu1, 2, \\ Meng-Zhong Liu1, $2 \bowtie$ \\ 1. State Key Laboratory of Oncology in South China, Guangdong Esophageal Cancer Institute;Collaborative Innovation Center for Cancer Medicine, \\ Guangzhou, China; \\ 2. Department of Radiation Oncology, Cancer Center, Sun Yat-sen University, Guangzhou, China; \\ 3. Imaging Diagnosis and Interventional Center, Cancer Center, Sun Yat-sen University, Guangzhou, China.
}

Note: The first two authors contributed equally to this work.

$\triangle$ Corresponding author: Dr. Meng-Zhong Liu, M.D., Department of Radiation Oncology, Cancer Center, Sun Yat-Sen University, No. 651DongfengEast Road, Guangzhou 510060, China. Phone: +86-20-87343492; Fax: +86-20-87343469; E-mail: liumengzhong@126.com

( ) Ivyspring International Publisher. This is an open access article distributed under the terms of the Creative Commons Attribution (CC BY-NC) license (https://creativecommons.org/licenses/by-nc/4.0/). See http://ivyspring.com/terms for full terms and conditions.

Received: 2017.05.22; Accepted: 2017.08.30; Published: 2017.09.20

\begin{abstract}
Purpose: To compare the clinical outcomes of induction chemotherapy (IC) followed by chemoradiotherapy (CRT) versus chemoradiotherapy alone in patients with locally advanced esophageal squamous cell carcinoma (ESCC).

Patients and methods: Between 2002 and 2015, 267 ESCC patients who received definitive CRT with docetaxel and cisplatin were enrolled in this study. Through a matched case-control study, 85 patients receiving IC before CRT were matched 1:1 to patients who received CRT alone, according to age, gender, performance status, tumor location, tumor length, and pretreatment TNM stage.

Results: The median overall survival (OS) in the IC group was significantly better than that in the CRT group ( 26.0 vs. 22.0 months), with 3 -year OS rates of $30.6 \%$ vs. $25.9 \%$, respectively $(P=0.028)$. However, IC plus CRT was associated with a significantly higher rate of grade 3-4 leukopenia than CRT alone $(P=0.048)$. The overall clinical response rate was $50.6 \%$ after IC in the IC group. The IC responder group showed significantly more favorable OS $(P=0.002)$ and progression-free survival $(P=0.001)$ compared with the IC non-responder group and the CRT group. Multivariate analysis revealed that age $\geq 60(P=0.003)$ and the addition of IC $(P=0.016)$ were independent prognostic factors that affected survival positively.

Conclusions: The addition of IC before CRT yielded satisfactory clinical outcomes and manageable toxicities. The combination of IC with CRT might be a promising treatment strategy to further improve systemic control and survival in ESCC. Prospective randomized trials are required to confirm the role of IC.
\end{abstract}

Key words: Esophageal squamous cell carcinoma, induction chemotherapy, definitive chemoradiotherapy, survival, toxicity.

\section{Introduction}

Esophageal cancer (EC) is the third most common cause of cancer-related death in China [1]. More than $50 \%$ of newly diagnosed EC cases are classified as locally advanced disease. Concurrent chemoradiotherapy (CRT) is recommended as the standard treatment for locally advanced unresectable
EC [2]. However, the prognosis for EC patients after definitive CRT remains poor, with local recurrence and distant metastasis being the major patterns of failure: the 5-year overall survival (OS) rate remains less than $20 \%$ [3].

In theory, the addition of induction 
chemotherapy (IC) before definitive CRT has the potential to achieve benefit in terms of a better response rate, early eradication of micrometastases, increased tumor radiosensitivity because of tumor shrinkage, and even overall survival [4]. Currently, several lines of evidence support the addition of IC to CRT in locally advanced esophageal squamous cell carcinoma (ESCC). In trial INT 0122, 38 patients with clinical Stage T1-4N0-1M0 ESCC received IC followed by CRT based on cisplatin combined with 5-fluorouracil (the PF regimen) were eligible, with $47 \%$ achieving a complete response (CR) and the median survival was 20 months. After IC, 90\% symptoms of dysphagia improved significantly [5]. Michel et al. reported that induction cisplatin-irinotecan followed by CRT without surgery for stage I-III esophageal cancer resulted in a complete clinical response rate of $58.1 \%$ and overall survival rates at 1 and 2 years of $62.8 \%$ and $27.9 \%$, respectively, in a multicenter phase II FFCD trial [6]. Akinori et al. found that IC responder patients showed an improved survival rate and no increase in severe toxicity in T4M0 esophageal cancer [7]. Moreover, Hironaga et al. conducted a prospective, multicenter phase I/II study and found that the 3-year survival rate was higher in the IC group compared with that in the control cohort [8].

The value of adding IC before definitive CRT in ESCC is still unclear because of the lack of prospective randomized trials. Thus we analyzed retrospectively the data of 267 patients receiving IC plus CRT or CRT alone, using the matched case-control method to compare the clinical outcomes. This study provided valuable effect assessment and evidence for future randomized controlled trials.

\section{Patients and Methods}

\section{Patients}

Between January 2002 and December 2015, 408 consecutive EC patients who underwent definitive CRT at the authors' institution were reviewed retrospectively. Criteria for inclusion were as follows: (1) histologically confirmed thoracic ESCC; (2) Eastern Cooperative Oncology Group performance status (PS) $\leq$ 2; (3) no distant organ metastasis; (4) no anticancer therapy history; (5) no concomitant or previous malignancy history; (6) unresectable or inoperable; (7) chemotherapy regimen comprising docetaxel and cisplatin (DP); (8) multidisciplinary evaluation before initiation of treatment; and (9) complete and retrievable clinical data. Overall, 85 patients fulfilling these criteria, who received IC before CRT, were defined as the study group. These patients were matched 1:1 to randomly selected patients who received CRT alone during the same study period, defined as the control group. The two groups were matched in a blinded fashion to the clinical outcomes, in accordance with the most relevant prognostic factors, including age (within 5 years), gender, PS score (0 vs. 1-2), tumor location (upper vs. middle vs. lower third), tumor length (within $3 \mathrm{~cm}$ ), and pretreatment TNM stage (III vs. IV). This study was approved by the institutional review board.

\section{Pretreatment Work-up}

Pretreatment work-up included physical examination, standard laboratory tests, a barium swallow test, endoscopy with biopsies, endoscopic ultrasonography (EUS), pulmonary function test, and thoracoabdominal computed tomography (CT). Bone scans and positron emission tomography (PET) were performed selectively. Staging was performed according to the sixth TNM staging system of the American Joint Committee on Cancer [9].

\section{Treatment}

All patients received concurrent chemotherapy with a three-weekly schedule of docetaxel $\left(60 \mathrm{mg} / \mathrm{m}^{2}\right.$ on day 1) plus cisplatin $\left(60-80 \mathrm{mg} / \mathrm{m}^{2}\right.$ on day 1$)$ or weekly schedule of docetaxel $\left(25 \mathrm{mg} / \mathrm{m}^{2}\right.$ on day 1$)$ plus cisplatin $\left(25 \mathrm{mg} / \mathrm{m}^{2}\right.$ on day 1$)$ for $5-7$ weeks. For the IC group, patients were treated with two cycles of docetaxel $\left(75 \mathrm{mg} / \mathrm{m}^{2}\right.$ on day 1) plus cisplatin $(75$ $\mathrm{mg} / \mathrm{m}^{2}$ on day 1) before CRT [10]. Dose adjustment was performed in the following chemotherapy cycle.

Radiation therapy was delivered by three-dimensional conformal radiotherapy or intensity-modulated radiotherapy with 6-8 MV X-ray. The gross tumor volume (GTV) was defined as the primary tumor and positive lymph nodes. The clinical target volume (CTV) comprised the GTV with a 3-cm margin in the cephalad and caudal directions, and a radial margin of $0.5-1.0 \mathrm{~cm}$. CTV also included the regional lymphatic regions. The planning target volume included the CTV with a 1-cm margin in all directions. A standard prescription dose of $60 \mathrm{~Gy}$ was delivered in 1.8-2.0 Gy fractions over 6-7 weeks.

\section{Toxicity and follow-up}

The toxicities of CRT were graded according to the National Cancer Institute Common Toxicity Criteria (version 3.0). Patients were followed up via physical examination, chest and abdominal CT, endoscopy with biopsies, and barium esophagography at 3-monthly intervals for the first two years, and every 6 months thereafter. The data were updated in December 2016.

\section{Statistical Analysis}

The clinical tumor response after IC was 
assessed by barium swallow and CT. CR was defined as no unequivocal soft tissue mass in the local region and all regional lymph nodes were less than $1.0 \mathrm{~cm}$ in the short axis. Partial response (PR) was defined as at least a $30 \%$ decrease in the sum of the diameters of the target lesions for over four weeks. Progressive disease (PD) was defined as at least a $20 \%$ increase in the sum of the diameters of the target lesions, or the appearance of one or more new lesions. Stable disease (SD) was defined as neither sufficient shrinkage to qualify for PR nor sufficient increase to qualify for PD.

Categorical variables were compared using the chi-square test or Fisher's exact test. The primary endpoint was OS, and the secondary endpoints were progression-free survival (PFS), distant metastasis-free survival (DMFS), and locoregional relapse-free survival (LRFS). OS was calculated from the diagnosis of ESCC until death or last follow-up. PFS was defined as the duration until locoregional recurrence or distant progression, last follow-up, or death. DMFS was defined as the time from treatment to the first distant metastasis, and LRFS was defined as the time from treatment to the first locoregional relapse. The Kaplan-Meier method was used to estimate the survival curves; the differences were compared using the log-rank test. A multivariate analysis using Cox proportional hazards survival regression analyses with stepwise regression (forward selection) for the whole cohort was conducted to evaluate the influence of the clinical variables on OS and PFS. All statistical analyses were performed using SPSS 19.0 software (SPSS Inc., Chicago, IL, USA) and Stata 12.0. A two-sided $P$-value $<0.05$ was considered statistically significant.

\section{Results}

\section{Patient and Tumor Characteristics}

The baseline characteristics of the 170 patients are listed in Table 1 . Within the whole series, the median age was 60 years (range, $47-70$ years), and the male to female ratio was 5.5:1. The median length of the primary tumor was $6 \mathrm{~cm}$ (range, 2.5-13.0 cm), and the majority of tumors were located in the middle third of the esophagus $(74.1 \%)$. Overall, 28 patients were classified as stage III (16.5\%) and 142 as stage IV $(83.5 \%)$. Forty-four patients were treated with three-dimensional conformal radiotherapy (3DCRT) and 126 received intensity-modulated radiation therapy (IMRT). The median radiation dose was 60 Gy (range, 50-70 Gy) in the whole cohort. Patient and disease-related characteristics were well balanced between the two treatment groups.

\section{Response to induction chemotherapy}

For patients in the IC group, 77 patients (90.6\%) had two cycles of IC, three (3.5\%) had one cycle, and 5 $(5.8 \%)$ had three cycles. After IC, CR was obtained in 0 patients $(0 \%)$, a PR in $43(50.6 \%)$, SD in $32(37.6 \%)$, and PD in 10 patients $(11.8 \%)$, respectively. The overall response rate $(\mathrm{CR}+\mathrm{PR})$ was $50.6 \%$.

Table 1. Patient and Tumor Characteristics

\begin{tabular}{|c|c|c|c|c|}
\hline Characteristic & $\begin{array}{l}\text { Total } \\
(n=170), \%\end{array}$ & $\begin{array}{l}\text { IC+CRT } \\
(n=85), \%\end{array}$ & $\begin{array}{l}\text { CCRT } \\
(n=85), \%\end{array}$ & $P$ \\
\hline \multicolumn{5}{|l|}{ Sex } \\
\hline Male & $144(84.7)$ & $72(84.7)$ & $72(84.7)$ & 1.000 \\
\hline Female & $26(15.3)$ & $13(15.3)$ & $13(15.3)$ & \\
\hline \multicolumn{5}{|l|}{ Age (years) } \\
\hline$<60$ & $107(62.9)$ & $51(60.0)$ & $56(65.9)$ & 0.427 \\
\hline$\geq 60$ & $63(37.1)$ & $34(40.0)$ & $29(34.1)$ & \\
\hline \multicolumn{5}{|l|}{ Smoking } \\
\hline Yes & 132(77.6) & $73(85.9)$ & $59(69.4)$ & 0.010 \\
\hline No & $38(22.4)$ & $12(14.1)$ & $26(30.6)$ & \\
\hline \multicolumn{5}{|l|}{ Drinking } \\
\hline Yes & $129(75.9)$ & $60(70.6)$ & $69(81.2)$ & 0.107 \\
\hline No & $41(24.1)$ & $25(29.4)$ & $16(18.8)$ & \\
\hline \multicolumn{5}{|l|}{ Weight loss } \\
\hline$<10 \%$ & $139(84.7)$ & $71(83.5)$ & $68(80.0)$ & 0.692 \\
\hline$\geq 10 \%$ & $31(15.3)$ & $14(16.5)$ & $17(20.0)$ & \\
\hline \multicolumn{5}{|l|}{ ECOG PS } \\
\hline 0 & $28(16.5)$ & $14(16.5)$ & $14(16.5)$ & 1.000 \\
\hline $1-2$ & $142(83.5)$ & $71(83.5)$ & $71(83.5)$ & \\
\hline \multicolumn{5}{|l|}{ Tumor location } \\
\hline Upper third & $32(18.8)$ & $16(18.8)$ & $16(18.8)$ & 1.000 \\
\hline Middle third & $126(74.1)$ & $63(74.1)$ & $63(74.1)$ & \\
\hline Lower third & $12(7.1)$ & $6(7.1)$ & $6(7.1)$ & \\
\hline \multicolumn{5}{|l|}{ Primary tumor length } \\
\hline$<6 \mathrm{~cm}$ & $68(40.0)$ & $29(42.6)$ & $39(45.9)$ & 0.117 \\
\hline$\geq 6 \mathrm{~cm}$ & $102(60.0)$ & $56(65.9)$ & $46(54.1)$ & \\
\hline \multicolumn{5}{|l|}{ Histologic grade } \\
\hline $\begin{array}{l}\text { Well/Moderately } \\
\text { differentiated }\end{array}$ & $109(64.1)$ & $56(65.9)$ & $53(62.4)$ & 0.631 \\
\hline Poorly differentiated & $61(35.9)$ & $29(34.1)$ & $32(37.6)$ & \\
\hline \multicolumn{5}{|l|}{ T stage } \\
\hline T1-2 & $9(5.3)$ & $4(4.7)$ & $5(5.9)$ & 0.732 \\
\hline T3-4 & $161(94.7)$ & $81(95.3)$ & $80(94.1)$ & \\
\hline \multicolumn{5}{|l|}{ N stage } \\
\hline No & $14(8.2)$ & $6(7.1)$ & $8(9.4)$ & 0.577 \\
\hline N1 & $156(91.8)$ & $79(92.9)$ & 77 (90.6) & \\
\hline \multicolumn{5}{|l|}{ M stage } \\
\hline M0 & $28(16.5)$ & $14(16.5)$ & $14(16.5)$ & 1.000 \\
\hline M1 & $142(83.5)$ & $71(83.5)$ & $71(83.5)$ & \\
\hline \multicolumn{5}{|c|}{ Pretreatment TNM stage } \\
\hline III & $28(16.5)$ & $14(16.5)$ & $14(16.5)$ & 1.000 \\
\hline IV & $142(83.5)$ & $71(83.5)$ & $71(83.5)$ & \\
\hline \multicolumn{5}{|c|}{ Radiotherapy technique } \\
\hline IMRT & $126(74.1)$ & $67(78.8)$ & $59(69.4)$ & 0.161 \\
\hline 3DCRT & $44(25.9)$ & $18(21.2)$ & $26(30.6)$ & \\
\hline \multicolumn{5}{|c|}{$\begin{array}{l}\text { Concurrent chemotherapy } \\
\text { regimen }\end{array}$} \\
\hline Weekly & $78(45.9)$ & $44(51.8)$ & $34(40.0)$ & 0.124 \\
\hline Three-weekly & $92(54.1)$ & $41(48.2)$ & $51(60.0)$ & \\
\hline
\end{tabular}

\section{Toxicity}

The incidences of grade 3-4 toxicities are summarized in Table 2. Grade 3-4 hematological toxicity occurred in $38.8 \%$ of patients receiving IC versus $28.2 \%$ of patients who received CRT alone $(P=$ 
0.317). The IC group had significantly higher proportions of grade 3-4 leucopenia than the CRT group $(P=0.048)$. The most commonly documented treatment-related acute toxicities were skin toxicity, fatigue, nausea, and esophagitis. There were no significant differences in the occurrence of non-hematological toxicities between the two groups. No treatment-related deaths were recorded.

Table 2. Treatment-related toxicity (NCICTC 3.0)

\begin{tabular}{llll}
\hline Event of grade $\geq \mathbf{3}$ & $\begin{array}{l}\text { IC+CRT } \\
(\boldsymbol{n}=\mathbf{8 5}), \boldsymbol{\%}\end{array}$ & $\begin{array}{l}\text { CCRT } \\
(\boldsymbol{n}=\mathbf{8 5}), \boldsymbol{\%}\end{array}$ & $\boldsymbol{P}$ \\
\hline $\begin{array}{l}\text { Hematological toxicity } \\
\text { Anemia }\end{array}$ & $1(1.2)$ & $2(2.4)$ & 1.000 \\
Leukopenia & $33(38.8)$ & $21(24.7)$ & 0.048 \\
Neutropenia & $28(32.9)$ & $20(23.5)$ & 0.173 \\
Thrombocytopenia & $8(9.4)$ & $5(5.9)$ & 0.387 \\
Non-hematological toxicity & & & \\
Nausea/vomiting & $8(9.4)$ & $5(5.9)$ & 0.387 \\
Diarrhea & $2(2.4)$ & $2(2.4)$ & 1.000 \\
Esophagitis & $6(7.1)$ & $5(5.9)$ & 0.755 \\
Pneumonitis & $3(3.5)$ & $2(2.4)$ & 0.650 \\
Skin toxicity & $13(15.3)$ & $16(18.8)$ & 0.541 \\
Weight loss & $7(8.2)$ & $8(9.4)$ & 0.787 \\
Fatigue & $17(20.0)$ & $13(15.3)$ & 0.421 \\
\hline
\end{tabular}

Abbreviations: NCICTC3.0: National Cancer Institute Common Toxicity Criteria (version 3.0)

\section{Follow-up and Survival}

In the original unmatched cohort of 267 patients, the median follow-up time was 26.0 months (8.0-99.0 months) for the IC group and 28.0 months (3.0-121.0 months) for the CRT group, respectively. The median OS for the whole cohort was 24 months (95\% confidence interval [CI], 18.1-29.9 months), with a 3-year OS rate of 28.2\%. In December 2016, 113 $(66.5 \%)$ patients had died and $57(33.5 \%)$ remained alive in the whole group. A total of $52(61.2 \%)$ patients had died and $33(38.8 \%)$ remained alive in the IC group, whereas, $61(71.8 \%)$ patients had died and 24 $(28.2 \%)$ remained alive in the CRT group. Among the 170 matched patients, 37 had locoregional recurrence only, 31 had distant metastasis only, 47 had concurrent locoregional and distant failure, and 55 had not developed recurrence. A total of 54 (63.5\%) patients had recurrence in the IC group, and 61 (71.8\%) in the CRT group. No statistically significant differences were observed between the IC group and the CRT group in terms of the survival rate $(P=0.144)$ and the recurrence rate $(P=0.251)$.

The median OS in the IC group was significantly longer compared with that in the CRT group (26.0 vs. 22.0 months), with 3 -year OS rates of $30.6 \%$ vs. $25.9 \%$, respectively $(P=0.028$, Figure $1 \mathrm{~A})$. Likewise, the 3-year PFS rate of the IC group was significantly higher than that of the CRT group ( $24.7 \%$ vs. $16.5 \%)$, with a median PFS of 24.0 vs. 16.0 months, respectively $(P=0.015$, Figure $1 \mathrm{~B})$.

Patients in the IC group were divided into two subgroups, including 43 IC responders $(C R+P R)$ and 42 non-responders (PD+SD). As shown in Figure 2, the IC responders were significantly better compared with the IC non-responders in terms of OS $(P=0.002)$, PFS $(P=0.001)$ and LRFS $(P=0.002)$, but not in DMFS $(P=0.116)$. No statistically significant differences were observed between the IC non-responder group and the CRT alone group in terms of OS $(P=0.963)$, PFS $(P=0.923)$, DMFS $(P=0.366)$, and LRFS $(P=$ $0.355)$.

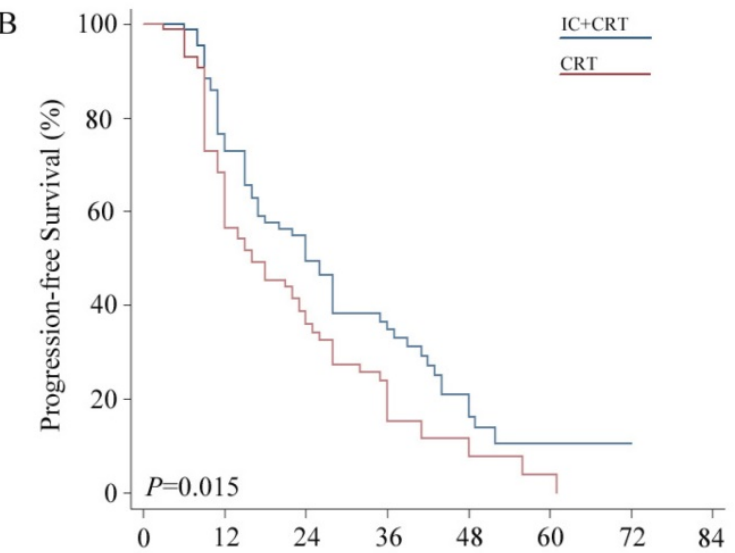

Number at risk

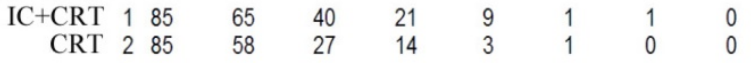

Figure 1. Kaplan-Meier survival curves for the induction chemotherapy plus by chemoradiotherapy (IC+CRT) group versus the CRT group. (A) Overall survival (OS); (B) Progression free survival (PFS). 
A

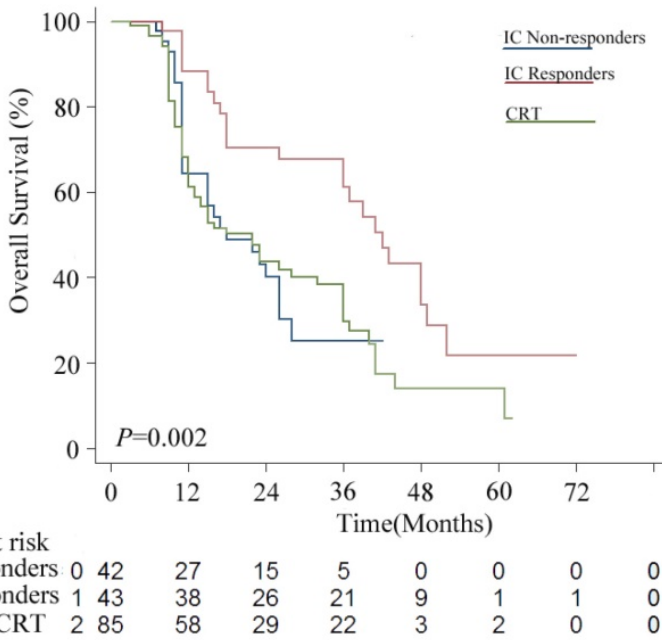

Number at risk IC Non-responders $0 \quad 42$

IC Responders 143 CRT 285

C

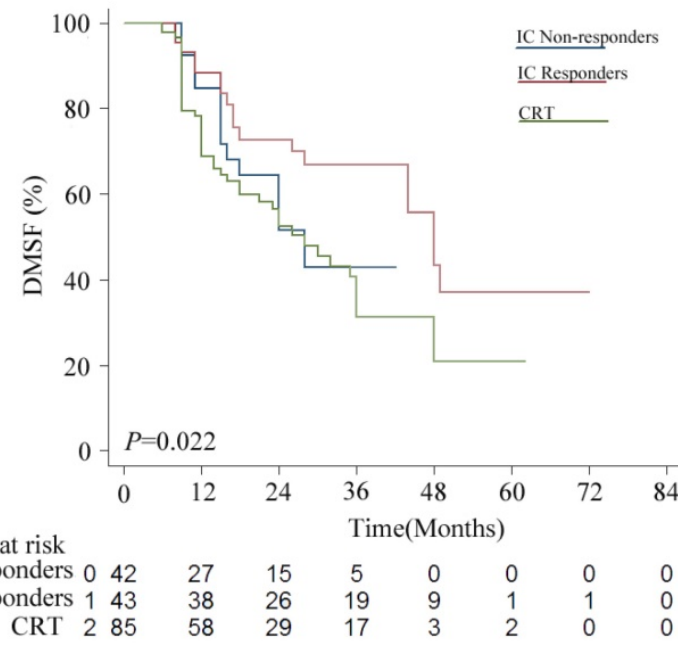

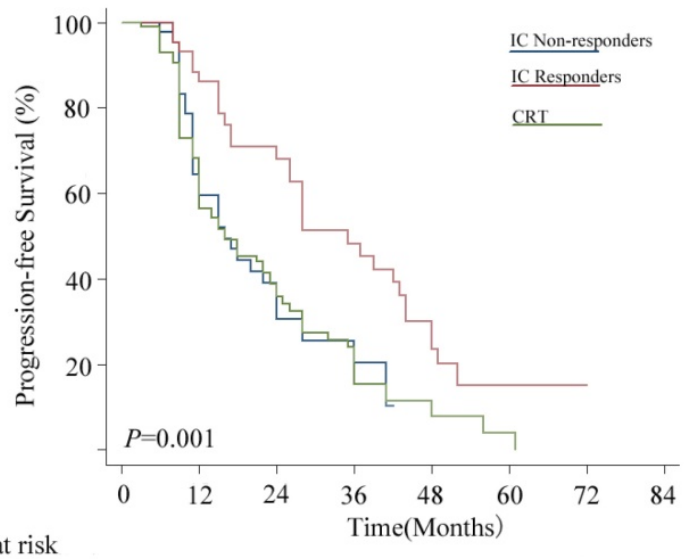

Number at risk

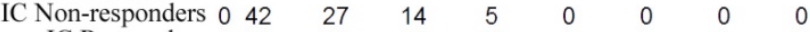

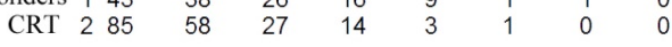

Number at risk

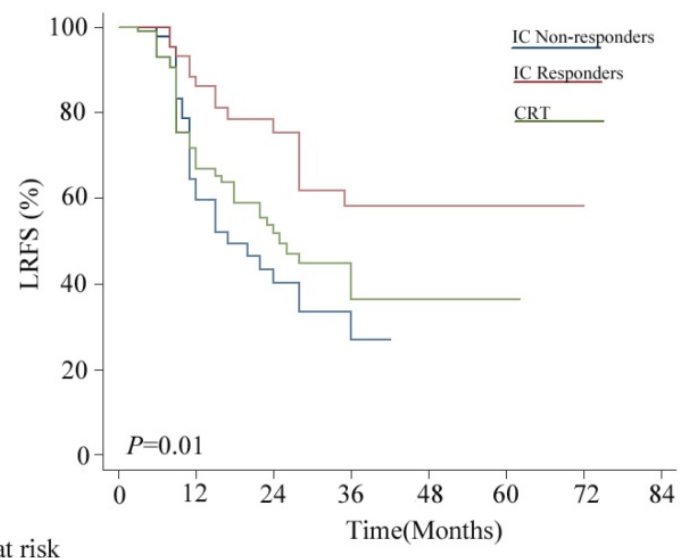

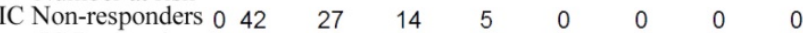

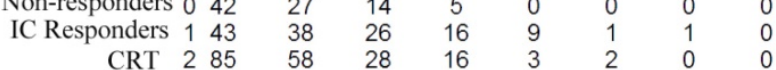

Figure 2. Kaplan-Meier survival curves for the induction chemotherapy (IC) non-responder group versus the IC responder group versus the chemoradiotherapy (CRT group). (A) Overall survival (OS); (B) Progression free survival (PFS); (C) distant metastasis-free survival (DMFS); (D) locoregional relapse-free survival (LRFS).

\section{Prognostic Analysis}

For the entire group, univariate analysis indicated that sex, smoking, drinking, weight loss before treatment, performance status, primary tumor location, primary tumor length, histologic grade, radiotherapy technique, and CRT regimen did not affect OS significantly (Table 3). Statistically significant factors or those approaching significance $(P<0.1)$ in the univariate analysis were subsequently included in the multivariate analysis, which showed that age $\geq 60(P=0.003)$ and the addition of IC $(P=$ $0.016)$ were independent prognostic factors that affected survival positively.

\section{Discussion}

Although the standard care for locally advanced esophageal cancer is definitive CRT, the long-term outcomes remain limited [11]. The efficacy and toxicity of IC before definitive CRT for ESCC patients have not been well documented previously. In the current study, a matched case-control method was used to adjust for differences in the baseline characteristics of the patients and to reduce selection bias, enabling an accurate comparison of the efficacies of these chemotherapy sequences. We found that patients who received IC plus CRT, especially the IC responders, had a significant survival advantage over patients receiving CRT alone.

The evidence supporting administration of IC before neoadjuvant CRT in esophageal cancer is controversial. Heta et al. analyzed retrospectively 119 esophageal cancer patients who underwent IC before neoadjuvant CRT and indicated that the addition of IC did not improve their pathological responses, OS, or DFS [12]. Ajani et al performed a prospective phase II randomized trial to compare IC followed by neoadjuvant CRT versus neoadjuvant CRT with oxaliplatin/fluorouracil regimens. However, the addition of IC produced only a non-significant increase in the pathological CR rate and did not prolong the OS [13]. In another randomized phase II 
trial, Doket et al. also reported that the addition of IC before neoadjuvant CRT failed to improve the pathological response and survival [14]. Above all, the addition of IC to neoadjuvant CRT failed to provide an obvious benefit in esophageal cancer. In contrast, in the present study, the OS in the IC group was significantly more favorable than that in the CRT alone group. There are several possible reasons for the difference. First, the majority of patients were esophageal adenocarcinoma in these studies, which is different from the histology of SCC in our study. Second, there were few patients with $\mathrm{T} 4$ or stage IV disease in the previous studies. It has been reported that patients with $\mathrm{T} 4 \mathrm{had}$ an incidence of perforation of $14-23 \%$ during CRT. The addition of IC before CRT might reduce the risk of perforation by decreasing the tumor volume before encountering severe esophagitis, which would benefit prolonged survival $[15,16]$. Akinori et al. found that IC for T4 esophageal cancer was effective to resolve severe dysphagia [7]. Moreover, $90 \%$ of the symptoms of dysphagia improved significantly after IC in the trial INT 0122 [5]. Therefore, IC might only be of benefit in some high-risk patients, such as ESCC with bulky tumors or stage IV disease.

Michael et al. revealed that the clinical tumor response to IC was the single independent prognostic factor for overall survival in patients who received IC followed by CRT, with or without surgery [17]. Similarly, Akinori et al. documented that the CR rate after CRT and the OS in the IC responder group were significantly better than those in the non-responder group $(P<0.01)$. Consistently, in our study, we found that the IC responders had significantly better survival at all end-points compared with the IC non-responders. In addition, no statistical differences in survival were observed between the IC non-responder group and the CRT group. Our data suggested that the IC responders might have a better CRT effect, prolonged OS, improved locoregional control, and reduced distant metastasis. Thus, it would be desirable to provide further insights into the potentially important role of distinguishing the IC responders from the whole group, or to identify potential biomarkers that might be beneficial in predicting chemoradiosensitivity and prognosis in ESCC.

Although the classical chemotherapy regimen for esophageal cancer patients is fluorouracil/cisplatin (PF), the prognosis is unsatisfactory [18]. Recently, there have been multiple reports about radiotherapy combined with docetaxel-based chemotherapy in esophageal carcinoma. Docetaxel is a taxoid that is derived semi-synthetically from the precursor 10-decacetyl baccatin III, which has a radiation-sensitizing effect in in vitro studies [19]. A randomized clinical study of 90 ESCC patients undergoing definitive CRT showed that the DP regimen yielded a better overall response rate and OS compared with PF (median OS: 43.2 vs. 22.3 months, $P<0.05$ ) [20]. Besides, a propensity score-matched analysis reported by Zhang $\mathrm{P}$ et al. indicated that CRT with DP induced better survival outcomes than PF $(P=0.009)$ [21]. In the present study, we also demonstrated that the DP regimen could produce encouraging results in ESCC, which is consistent with previous reports.

Table 3. Univariate and multivariate analysis of prognostic factors related to survival $(n=170)$

\begin{tabular}{|c|c|c|c|c|c|}
\hline \multirow[t]{2}{*}{ Factor } & \multirow[t]{2}{*}{ No. } & \multicolumn{2}{|l|}{ Univariate } & \multicolumn{2}{|l|}{ Multivariate } \\
\hline & & $\begin{array}{l}\text { 3-year OS } \\
(\%)\end{array}$ & $P$-value & $\begin{array}{l}\text { Hazards ratio } \\
(95 \% \mathrm{CI})\end{array}$ & $P$-value \\
\hline \multicolumn{6}{|l|}{ Sex } \\
\hline Male & 144 & 27.8 & 0.982 & & \\
\hline Female & 26 & 30.8 & & & \\
\hline \multicolumn{6}{|l|}{ Age (years) } \\
\hline$<60$ & 107 & 36.4 & 0.013 & $\begin{array}{l}1.796 \\
(1.216-2.652)\end{array}$ & 0.003 \\
\hline$\geq 60$ & 63 & 14.3 & & & \\
\hline \multicolumn{6}{|l|}{ Smoking } \\
\hline Yes & 132 & 28.8 & 0.450 & & \\
\hline No & 38 & 26.3 & & & \\
\hline \multicolumn{6}{|l|}{ Drinking } \\
\hline Yes & 129 & 27.9 & 0.308 & & \\
\hline No & 41 & 29.3 & & & \\
\hline \multicolumn{6}{|l|}{ Weight loss } \\
\hline$<10 \%$ & 139 & 27.3 & 0.482 & & \\
\hline$\geq 10 \%$ & 31 & 32.3 & & & \\
\hline \multicolumn{6}{|l|}{ Performance status } \\
\hline 0 & 28 & 28.6 & 0.641 & & \\
\hline $1-2$ & 142 & 28.2 & & & \\
\hline \multicolumn{6}{|l|}{ Tumor location } \\
\hline Upper third & 32 & 31.3 & 0.500 & & \\
\hline Middle third & 126 & 28.6 & & & \\
\hline Lower third & 12 & 16.7 & & & \\
\hline \multicolumn{6}{|l|}{ Primary tumor length } \\
\hline$<6 \mathrm{~cm}$ & 68 & 30.8 & 0.863 & & \\
\hline$\geq 6 \mathrm{~cm}$ & 102 & 26.5 & & & \\
\hline \multicolumn{6}{|l|}{ Histologic grade } \\
\hline $\begin{array}{l}\text { Well/Moderately } \\
\text { differentiated }\end{array}$ & 109 & 27.5 & 0.398 & & \\
\hline Poorly differentiated & 61 & 29.5 & & & \\
\hline \multicolumn{6}{|l|}{$\begin{array}{l}\text { Pretreatment TNM } \\
\text { stage }\end{array}$} \\
\hline III & 28 & 46.4 & 0.054 & $\begin{array}{l}1.780 \\
(1.047-3.025)\end{array}$ & 0.330 \\
\hline IV & 142 & 24.6 & & & \\
\hline \multicolumn{6}{|l|}{ Therapy regimen } \\
\hline IC+CRT & 85 & 30.6 & 0.028 & $\begin{array}{l}1.592 \\
(1.092-2.322)\end{array}$ & 0.016 \\
\hline CRT & 85 & 25.9 & & & \\
\hline \multicolumn{6}{|c|}{ Radiotherapy technique } \\
\hline IMRT & 126 & 27.0 & 0.715 & & \\
\hline 3DCRT & 44 & 31.8 & & & \\
\hline \multicolumn{6}{|c|}{$\begin{array}{l}\text { Concurrent } \\
\text { chemotherapy regimen }\end{array}$} \\
\hline Weekly & 78 & 32.1 & 0.231 & & \\
\hline Three-weekly & 92 & 25.0 & & & \\
\hline
\end{tabular}


However, induction chemotherapy might have adverse effects on patient tolerance to subsequent concurrent CRT. In the phase II randomized trial of RTOG 0113, Ajani et al. reported that adding IC was associated with considerable morbidity $(80 \%$ rate of grade 3 or 4 toxicities) [22]. Michel et al. reported that grade 3 or 4 toxicities were observed in $23.3 \%$ and $64.5 \%$ of patients, of which $57.1 \%$ and $58.1 \%$ were caused by hematoxicity during IC or CRT with cisplatin/irinotecan [6]. In the current study, grade 3-4 hematological toxicities were observed in 38.8\% and $28.2 \%$ of patients receiving IC+CRT or CRT alone, respectively. The IC group had significantly higher proportions of grade 3-4 leucopenia than the CRT group $(P=0.048)$. However, although the risk of hematological toxicities in the IC group was higher than the CRT group, the toxicities were manageable and could be mitigated by preventive measures, suggesting that the combination of IC and CRT based on the DP regimen was well tolerated.

This study had several limitations. First, the study was limited by its retrospective nature and the fact it was conducted in a single institution. Second, the chemotherapy regimen included weekly and three-weekly regimens. However, the chemotherapy regimen did not affect the OS significantly in the univariate analysis, and thus had limited effects on our results.

\section{Conclusions}

Our data demonstrated that a combination of IC and CRT might be a promising treatment strategy to further improve systemic control and survival of locally advanced ESCC. In addition, the results suggested that the IC responders have a better chemoradiotherapy effect, prolonged OS, improved locoregional control, and reduced distant metastasis. Prospective, randomized controlled trials are required to further confirm the role of IC.

\section{Acknowledgments}

This work was supported by grants from the National Natural Science Foundation of China (NSFC81272487).

\section{Competing Interests}

The authors have declared that no competing interest exists.

\section{References}

1. Chen WR, Zheng PD, Baade S, et al. Cancer statistics in China. CA Cancer J Clin. 2015; 66:115-132.

2. Cooper JS, Guo MD, Herskovic A, et al. Chemoradiotherapy of locally advanced esophageal carcinoma, long-term follow-up of a prospective randomized trial (RTOG 85-01). JAMA. 1999;281: 1623-1627.

3. Liao Z, Cox JD, Komaki R. Radiochemotherapy of esophageal cancer. J Thorac Oncol. 2007; 2:553-68.
4. Hui EP, Ma BB, Leung SF, et al. Randomized phase II trial of concurrent cisplatin-radiotherapy with or without neoadjuvant docetaxel and cisplatin in advanced nasopharyngeal carcinoma. J Clin Oncol. 2009; 27:242-9.

5. Minsky BD, Neuberg D, Kelsen DP, et al. Final report of Intergroup Trial 0122 (ECOG PE-289, RTOG 90-12): Phase II trial of neoadjuvant chemotherapy plus concurrent chemotherapy and high-dose radiation for squamous cell carcinoma of the esophagus. Int J Radiat Oncol Biol Phys. 1999; 43:517-23.

6. Michel P, Adenis A, Di Fiore F, et al. Induction cisplatin-irinotecan followed by concurrent cisplatin-irinotecan and radiotherapy without surgery in oesophageal cancer: multicenter phase II FFCD trial. Br J Cancer. 2006; 95:705-9.

7. Akinori M, Michitaka H, Yousuke I, et al. Induction chemotherapy followed by chemoradiotherapy for T4M0 esophageal cancer. Esophagus. 2011; 8:31-37

8. Satake $\mathrm{H}$, Tahara $\mathrm{M}$, Mochizuki S, et al. A prospective, multicenter phase I/II study of induction chemotherapy with docetaxel, cisplatin and fluorouracil (DCF) followed by chemoradiotherapy in patients with unresectable locally advanced esophageal carcinoma. Cancer Chemother Pharmacol. 2016; 78:91-9

9. Sobin LH, Wittekind Ch (eds). TNM Classification of Malignant Tumors, 6th edition. Hoboken, NJ: John Wiley \& Sons 2002.

10. Van Meerten E, van der Gaast A. Systemic treatment for oesophageal cancer. Eur J Cancer. 2005; 41:664-72.

11. Day FL, Leong T, Ngan S, et al. Phase I trial of docetaxel, cisplatin and concurrent radical radiotherapy in locally advanced oesophageal cancer. $\mathrm{Br} \mathrm{J}$ Cancer. 2011; 104:265-271.

12. Javeri H, Arora R, Correa AM, et al. Influence of Induction Chemotherapy and Class of Cytotoxics on Pathologic Response and Survival After Preoperative Chemoradiation in Patients With Carcinoma of the Esophagus. Cancer. 2008; 113:1302-8.

13. Ajani JA, Xiao L, Roth JA, et al. A phase II randomized trial of induction chemotherapy versus no induction chemotherapy followed by preoperative chemoradiation in patients with esophageal cancer. Ann Oncol. 2013;24:2844-9

14. Yoon DH, Jang G, Kim JH, al. Randomized phase 2 trial of S1 and oxaliplatin-based chemoradiotherapy wit $\mathrm{h}$ or without induction chemotherapy for esophageal cancer. Int J Radiat Oncol Biol Phys. 2015; 91:489-96.

15. Ohtsu A, Boku N, Muro K, et al. Definitive chemoradiotherapy for T4 and/or M1 lymph node squamous cell carcinoma of the esophagus. J Clin Oncol. 1999; 17:2915-2921.

16. Shinoda M, Ando N, Kato K, et al. Randomized study of low-dose versus standard-dose chemoradiotherapy for unresectable esophageal squamous cell carcinoma (JCOG0303). Cancer Sci. 2015; 106:407-412.

17. Stahl M, Stuschke M, Lehmann N, et al. Chemoradiation with and without surgery in patients with locally advanced squamous cell carcinoma of the esophagus. J Clin Oncol. 2005; 23:2310-7.

18. Ishida K, Ando N, Yamamoto S, et al. Phase - study of cisplatin and 5fluorouracil with concurrent radiotherapy in advanced squamous cell carcinoma of the esophagus: A Japan Esophageal Oncology Group (JEOG)/ Japan Clinical Oncology Group trial (JCOG9516). Jpn J Clin Oncol. 2004; 34:615-619.

19. Mason K A, Hunter N R, Milas M, et al. Docetaxel enhances tumor radioresponse in vivo. Clin Cancer Res. 1997; 3:2431-8.

20. Zhao T, Chen $\mathrm{H}$ and Zhang T. Docetaxel and cisplatin concurrent with radiotherapy versus 5 -fluorouracil and cisplatin concurrent with radiotherapy in treatment for locally advanced oesophageal squamous cell carcinoma: a randomized clinical study. Med Oncol. 2012; 29:3017-3023.

21. Zhang $\mathrm{P}, \mathrm{Xi} \mathrm{M}, \mathrm{Li} \mathrm{QQ}$, et al. Concurrent cisplatin and 5-fluorouracil versus concurrent cisplatin and docetaxel with radiotherapy for esophageal squamous cell carcinoma: a propensity score-matched analysis. Oncotarget. 2016; 7:44686-44694.

22. Ajani JA, Winter K, Komaki R, et al. Phase II randomized trial of two nonoperative regimens of induction chemotherapy followed by chemoradiation in patients with localized carcinoma of the esophagus: RTOG 0113. J Clin Oncol. 2008; 26:4551-6. 